\title{
Archivos \\ de Cardiología de México

\section{Beneficio de la implementación de un sistema de atención en red sobre las mujeres con infarto agudo de miocardio con elevación del segmento ST}

\author{
Diego Fernández-Rodríguez ${ }^{\mathrm{a}, \mathrm{b}}$, Xavier Freixa ${ }^{\mathrm{a}, *}$, Gizem Kasa ${ }^{\mathrm{a}}$, \\ Ander Regueiro ${ }^{\mathrm{a}, \mathrm{c}}$, Joaquim Cevallos ${ }^{\mathrm{a}}$, Marco Hernández ${ }^{\mathrm{a}}$, \\ Salvatore Brugaletta ${ }^{a}$, Victoria Martín-Yuste ${ }^{a}$, Manel Sabaté ${ }^{a, c}$ \\ y Mónica Masotti ${ }^{\text {a }}$ \\ a Servicio de Cardiología, Hospital Clínic, Barcelona, España \\ b Servicio de Cardiología, Hospital Nuestra Señora de Candelaria, Santa Cruz de Tenerife, España \\ c Comité Directivo, Iniciativa Stent for Life, España
}

Recibido el 21 de marzo de 2014; aceptado el 22 de diciembre de 2014

\author{
PALABRAS CLAVE \\ Género; \\ Infarto agudo de \\ miocardio con \\ elevación del \\ segmento ST; \\ Angioplastia \\ primaria; \\ Red
}

\begin{abstract}
Resumen
Objetivo: El sistema de atención en red por infarto con elevación del segmento ST «Codi Infart» se implementó en Cataluña (España) en junio de 2009. El objetivo del estudio fue evaluar el beneficio de la instauración del Codi Infart para las mujeres atendidas en nuestra institución. Método: Las mujeres referidas para angioplastia primaria se dividieron en 2 grupos de acuerdo con el Codi Infart: grupo no-Codi Infart (enero de 2003 a mayo de 2009) y grupo Codi Infart (junio de 2009 a diciembre de 2012); y se compararon lugar de procedencia, periodos, tratamientos recibidos y tasa de eventos cardiovasculares mayores definida como muerte por todas las causas, reinfarto o accidente cerebrovascular durante el ingreso, a 30 y 180 días.

Resultados: De una población total de 2,426 pacientes, 501 (20.7\%) eran mujeres. De ellas, 186 mujeres (2,09 casos/mes) pertenecían al grupo no-Codi Infart y 315 mujeres (10,16 casos/mes) al grupo Codi Infart. El porcentaje de mujeres atendidas aumentó desde la introducción del Codi Infart(22.2\% vs. $18.5 \%, p=0.028)$. Además, inicialmente el grupo Codi Infart presentó mayor porcentaje de mujeres atendidas fuera de nuestra institución ( $84.1 \%$ vs. $16.7 \%, p<0.001)$, y menores tiempos totales de isquemia $(220$ [155-380] vs. 272 [196-456], $p=0.003)$, pero no se detectaron diferencias en eventos cardiovasculares mayores a 180 días $(14.2 \%$ vs. $15.6 \%$, $p=0.692$ ).
\end{abstract}

\footnotetext{
* Autor para correspondencia. Hospital Clínic, Villarroel 170, 08035, Barcelona, España. Correo electrónico: FREIXA@clinic.ub.es (X. Freixa).
} 
Conclusiones: La instauración del Codi Infart permitió aumentar de manera notable la tasa y el porcentaje de mujeres con infarto de miocardio con elevación del segmento ST tratadas mediante angioplastia primaria y reducir los tiempos totales de isquemia.

(c) 2014 Instituto Nacional de Cardiología Ignacio Chávez. Publicado por Masson Doyma México S.A. Todos los derechos reservados.

\section{KEYWORDS}

Gender;

ST-segment elevation

myocardial

infarction;

Primary percutaneous

coronary

intervention;

Network

\section{Benefit of the implementation of a ST-segment elevation myocardial infarction network on women}

\begin{abstract}
Objective: The ST-segment elevation myocardial infarction network "Codi Infart" was implemented in Catalonia (Spain) in June 2009. The objective of this study was to evaluate the impact of the implementation of the Codi Infart on women.

Method: Women referred for primary percutaneous coronary intervention, were divided into two groups according to Codi Infart: Non-Codi Infart group (January 2003 to May 2009) and Codi Infart group (June 2009 to December 2012). Place of first medical contact, time intervals in diagnosis and treatment, treatments received and rate of major cardiovascular adverse events defined as all-cause death, reinfarction or stroke in-hospital, at 30 and 180 days were compared. Results: From a total population of 2,426 patients, 501 (20.7\%) were women. One-hundred eighty-six women (2.09 cases/month) belonged to Non-Codi Infart group and 315 women (10.16 cases/month) to Codi Infart group. The percentage of women attended increased since the introduction of $\mathrm{Cl}(22.2 \%$ vs. $18.5 \%, P=.028)$. In addition, the Codi Infart group had a higher percentage of women initially attended outside our institution $(84.1 \%$ vs. $16.7 \%, P<.001)$, and lower total ischemia time (220 [155-380] vs. 272 [196-456], $P=.003)$. However, no differences in 180-day major cardiovascular adverse events were detected $(14.2 \%$ vs. $15.6 \%, P=.692)$. Conclusions: The implementation of the major cardiovascular adverse events allowed to increase the rate and the percentage of women with ST-segment elevation myocardial infarction treated with primary percutaneous coronary intervention and reducing total ischemic time. (C) 2014 Instituto Nacional de Cardiología Ignacio Chávez. Published by Masson Doyma México S.A. All rights reserved.
\end{abstract}

\section{Introducción}

El infarto agudo de miocardio con elevación del segmento ST (IAMCEST) es uno de los principales problemas de salud pública, tanto en la población masculina como en la femenina $^{1,2}$. Por lo general las mujeres consultan de manera tardía y el diagnóstico es más complicado, ya que presentan mayor tasa de síntomas atípicos que los hombres ${ }^{3}$.

La angioplastia primaria (AP) es la terapia de reperfusión recomendada en el IAMCEST, siempre que se realice en los 120 primeros minutos después del primer contacto médico $(P C M)$. En mujeres, está recomendación es especialmente importante $^{1}$ ya que la AP mejora la supervivencia y reduce las complicaciones hemorrágicas de la trombolisis en mayor grado que en los hombres ${ }^{4-6}$. Asimismo, cuando las mujeres reciben una terapia de reperfusión efectiva mediante AP tienen el mismo pronóstico que los hombres ${ }^{4}$. Además, la transferencia de los pacientes desde hospitales sin posibilidad de realizar AP las $24 \mathrm{~h}$ del día, los 7 días de la semana (24/7) hacia hospitales con posibilidad de AP 24/7 se ha demostrado como una estrategia factible y segura, siendo avalada en múltiples estudios ${ }^{7-11}$.

Por estas razones, la implantación de las redes de atención al IAMCEST ha contribuido a extender ampliamente la reperfusión mediante $\mathrm{AP}$ en el periodo recomendado por las guías de práctica clínica ${ }^{1}$. Sin embargo, existen pocos datos sobre cómo las redes han modificado el tratamiento y el pronóstico de los IAMCEST en las mujeres ${ }^{12,13}$.

El objetivo de nuestro estudio fue analizar el beneficio de la implantación de las redes de atención al IAMCEST en la población femenina en una región de España.

\section{Métodos}

\section{Sistema de atención en red al infarto: «Codi Infart»}

En la etapa previa a la implantación del sistema regional catalán de atención al IAMCEST («Codi Infart» $[\mathrm{CI}])^{9,14}$, la mayor parte de los pacientes que recibieron reperfusión percutánea en muestro centro fueron quienes acudieron al servicio de urgencias de nuestro hospital, así como los pacientes que presentaban un IAMCEST estando ya previamente hospitalizados en nuestra institución. También recibieron terapia de reperfusión aquellas pacientes que presentaban contraindicaciones para la trombolisis, aquellas con ausencia de criterios de reperfusión después de la trombolisis y las que presentaban un choque cardiogénico 
enviadas de otros centros hospitalarios sin AP 24/7. Sin embargo, no había una estrategia estandarizada de sectorización del territorio, ni de priorización en los traslados ni de contacto sistemático entre el «Sistema d'Emergències Mèdiques» del Servicio Catalán de la Salud y nuestro centro. Además, tampoco existía una estrategia de pretratamiento de los pacientes referidos para AP.

El $\mathrm{Cl}^{9,14}$ se implantó en junio de 2009 para atender a la población de la comunidad autónoma de Cataluña, cubriendo una población de 7.5 millones de habitantes. La comunidad fue dividida en función de aspectos territoriales y de los recursos disponibles para realizar la terapia de reperfusión más adecuada a cada individuo. El «Sistema d'Emergències Mèdiques» cubría todo el territorio de Cataluña y se encargaba de coordinar todo el proceso de atención de pacientes hasta que los mismos ingresaban en un centro con disponibilidad de AP. Durante el periodo del estudio, el $\mathrm{Cl}$ se componía de 10 centros con capacidad de AP, 5 de los cuales contaban con un servicio 24/7.

Nuestra institución, el Hospital Clínic de Barcelona, disponía de capacidad para realizar AP 24/7 y se encargaba de la atención de las siguientes áreas sanitarias: Barcelona Esquerra, Vallés Occidental y Osona (aproximadamente 1,6 millones de habitantes y $1,840 \mathrm{~km}^{2}$ ).

Después de la implantación del $\mathrm{Cl}$, todos los pacientes con IAMCEST que ingresaban directamente en nuestro centro recibían AP. Los pacientes de nuestra área sanitaria que ingresaban en hospitales sin capacidad para realizar AP, en centros de atención primaria o tratados inicialmente por el «Sistema d'Emergències Mèdiques», fueron transferidos a nuestro centro según su perfil de riesgo y si el retraso esperado para la AP era inferior a $120 \mathrm{~min}$. De lo contrario, se administraba tratamiento trombolítico ${ }^{14}$. Por protocolo, cuando se decidía la realización de la AP, los pacientes recibían ácido acetilsalicílico $300 \mathrm{mg}$, clopidogrel $600 \mathrm{mg}$ y heparina no fraccionada 5,000 UI. Tras la AP, el tratamiento de prevención secundaria se prescribía de acuerdo con la práctica médica estándar ${ }^{1,2}$, y una vez estabilizados, los pacientes eran retornados a sus centros de referencia ${ }^{14}$.

El equipo de hemodinámica de guardia, para asegurar una organización eficiente en la provisión de la AP en el menor tiempo posible, estaba compuesto por 2 cardiólogos intervencionistas y 2 enfermeros especializados en hemodinámica en cada guardia localizada. Disponía, asimismo, de un único número telefónico para activación de todos sus miembros, y sus miembros se comprometían a estar en el laboratorio de hemodinámica en los primeros 20 min después de activación ${ }^{1,9,14}$.

\section{Población del estudio y seguimiento clínico}

Entre enero de 2003 y diciembre de 2012, todas las pacientes con IAMCEST atendidas consecutivamente en nuestro hospital para AP primaria o de rescate se incluyeron en nuestro registro. Se recopilaron prospectivamente las características clínicas y angiográficas de las pacientes, y se realizó un seguimiento clínico a los 30 y a los 180 días, mediante visita clínica o entrevista telefónica. Asimismo, todas las pacientes firmaron un consentimiento informado y el estudio se llevó a cabo en conformidad con la Declaración de Helsinki.

\section{Criterios de valoración y definiciones}

El criterio de valoración principal del estudio fue la tasa de eventos cardiovasculares adversos mayores, definida como la combinación de muerte por todas las causas, reinfarto o accidente cerebrovascular a 180 días.

Los criterios de valoración clínicos secundarios del estudio fueron: eventos cardiovasculares adversos mayores, muerte por cualquier causa, reinfarto o accidente cerebrovascular intrahospitalarios; eventos cardiovasculares adversos mayores, muerte por cualquier causa, reinfarto o accidente cerebrovascular a 30 días; y muerte por cualquier causa, reinfarto o accidente cerebrovascular a 180 días.

También se evaluaron otros criterios de valoración secundarios relacionados con la atención médica, tales como el lugar del PCM; los siguientes intervalos de atención médica: inicio de los síntomas-PCM, PCM-apertura de arteria, e inicio de los síntomas-apertura de arteria (tiempo total de isquemia) $)^{1,15}$; y los tratamientos recibidos por las pacientes. El PCM se definió como el lugar en el que el paciente fue evaluado inicialmente, por un médico o por cualquier otro tipo de personal médico, ya sea en el ámbito hospitalario o prehospitalario ${ }^{15}$. La tasa mensual de infartos tratados se definió como el número total de IAMCEST tratados dividido por el número de meses dentro de cada grupo de análisis.

Todas las variables de valoración se analizaron de acuerdo con la presencia o la ausencia del $\mathrm{Cl}$ (no- $\mathrm{Cl}$ vs. $\mathrm{Cl}$ ).

\section{Análisis estadístico}

Se exploró la normalidad de las variables continuas mediante la prueba de Kolmogorov-Smirnov. Las variables continuas distribuidas normalmente se expresaron como media (desviación estándar) y las variables continuas no distribuidas normalmente se expresaron como mediana (rango intercuartílico), y finalmente fueron comparadas mediante la prueba de T-student, de Mann-Whitney o de Kruskal-Wallis, según procediese. Las variables categóricas se expresaron como porcentaje y se compararon con la prueba de Ji-cuadrado.

Todos los análisis estadísticos se llevaron a cabo con el paquete SPSS 20.0 (SPSS Inc., Chicago, Illinois, EE. UU.) y para todos ellos se consideró significativo un valor de $p$ bilateral $<0.05$.

\section{Resultados}

Entre enero de 2003 y diciembre de 2012, un total de 2,426 pacientes que cumplían criterios de IAMCEST fueron atendidos en el laboratorio de hemodinámica del Hospital Clínic de Barcelona, de los cuales 501 (20.7\%) fueron mujeres. En el análisis por periodos se observó que en el tiempo previo a la implementación del $\mathrm{Cl}$ (enero de 2003 a mayo de 2009), se atendieron 1,005 pacientes, mientras que en el periodo posterior a la instauración del $\mathrm{Cl}$ (junio de 2009 a diciembre de 2012), se atendieron 1,421. Además se observó un aumento significativo del porcentaje de mujeres atendidas al pasar de $186(18.5 \%)$ antes del $\mathrm{Cl}$ a 315 (22.2\%) después de la introducción del $\mathrm{Cl}(18.5 \%$ vs. $22.2 \%, \mathrm{p}=0.028)$ (tabla 1$)$. 
Tabla 1 Características basales

\begin{tabular}{|c|c|c|c|}
\hline Población total & No- $\mathrm{Cl}(n=1,005)$ & $\mathrm{Cl}(\mathrm{n}=1,421)$ & Valor de $\mathrm{p}$ \\
\hline Mujeres, n (\%) & $186(18.5 \%)$ & $315(22.2 \%)$ & 0.028 \\
\hline Características de la población femenina & Mujeres no- $\mathrm{Cl}(\mathrm{n}=186)$ & Mujeres $\mathrm{Cl}(\mathrm{n}=315)$ & \\
\hline Edad (años), media (DE) & $68.4(13.1)$ & $71.2(13.1)$ & 0.021 \\
\hline Hipertensión arterial, $n$ (\%) & $113(60.8)$ & $212(67.3)$ & 0.138 \\
\hline Dislipidemia, $n(\%)$ & $81(43.5)$ & $141(44.8)$ & 0.792 \\
\hline Diabetes mellitus, $n(\%)$ & $52(28)$ & $85(27)$ & 0.813 \\
\hline Tabaquismo actual, $n$ (\%) & $48(25.8)$ & $61(19.4)$ & 0.091 \\
\hline Infarto de miocardio previo, $n$ (\%) & $18(9.7)$ & $28(8.9)$ & 0.768 \\
\hline Intervención coronaria previa, $n$ (\%) & $10(5.4)$ & $18(5.7)$ & 0.874 \\
\hline Cirugía coronaria previa, $n(\%)$ & $3(1.6)$ & $2(0.6)$ & 0.287 \\
\hline Vaso culpable, $n(\%)$ & & & 0.064 \\
\hline DA & $90(48.4)$ & $131(41.6)$ & \\
\hline$C D$ & $69(37.1)$ & $119(37.8)$ & \\
\hline $\mathrm{CX}$ & $24(12.9)$ & $61(19.4)$ & \\
\hline $\mathrm{TCl}$ & $1(0.5)$ & $2(0.1)$ & \\
\hline By-pass & $2(1.1)$ & $2(0.1)$ & \\
\hline Cantidad de vasos enfermos, media (DE) & $1.51(0.69)$ & $1.53(0.80)$ & 0.759 \\
\hline Killip al ingreso, $n(\%)$ & & & 0.699 \\
\hline 1 & $130(69.9)$ & $228(73.1)$ & \\
\hline II & $23(12.4)$ & $29(9.3)$ & \\
\hline III & $13(7)$ & $19(6.1)$ & \\
\hline IV & $20(10.8)$ & $36(11.5)$ & \\
\hline Flujo TIMI inicial, $n$ (\%) & & & 0.003 \\
\hline 0 & $105(57.1)$ & $212(67.3)$ & \\
\hline 1 & $33(17.9)$ & $39(12.4)$ & \\
\hline 2 & $25(13.6)$ & $18(5.7)$ & \\
\hline 3 & $21(11.4)$ & $46(14.6)$ & \\
\hline Flujo TIMI final, $n(\%)$ & & & 0.077 \\
\hline 0 & $8(4.3)$ & $18(5.7)$ & \\
\hline 1 & $4(2.2)$ & $4(1.3)$ & \\
\hline 2 & $21(11.4)$ & $17(5.4)$ & \\
\hline 3 & $152(82.2)$ & $276(87.6)$ & \\
\hline
\end{tabular}

CD: coronaria derecha; Cl: grupo posterior a la implantación del «Codi Infart»; CX: circunfleja; DA: descendente anterior; DE: desviación estándar; No-Cl: grupo previo a la implantación del «Codi Infart»; TCl: tronco común izquierdo.

\section{Características basales}

Las características basales en ambos grupos de mujeres se presentan en la tabla 1. La tasa mensual de mujeres con IAMCEST en el grupo no- $\mathrm{Cl}$ ascendía a 2.09 casos/mes; y en el grupo $\mathrm{Cl}$ a 10.16 casos/mes.

Cabe destacar que la edad en el grupo $\mathrm{Cl}$ era superior a la del grupo no- $\mathrm{Cl}(68.4 \pm 13,1$ vs. $71.2 \pm 13.1, \mathrm{p}=0.021)$, y el flujo TIMI inicial era inferior en el grupo $\mathrm{Cl}$ en comparación con el grupo no- $\mathrm{Cl}$. No se observaron otras diferencias entre ambos grupos.

\section{Procedencia de los pacientes, periodos y tratamiento}

Los datos que comparan la procedencia, los tiempos de atención y los tratamientos administrados entre los 2 grupos se presentan en la tabla 2.

Es de destacar que el lugar del PCM fue significativamente diferente entre ambos grupos. En el grupo no- $\mathrm{Cl}$, la fuente de los pacientes era predominantemente nuestra institución; mientras que en el grupo $\mathrm{Cl}$, la mayoría de los pacientes fueron referidos desde hospitales sin AP 24/7 y por el «Sistema d'Emergències Mèdiques».

Se observaron diferencias relacionadas con los periodos. El grupo $\mathrm{Cl}$ presentaba menor intervalo inicio de síntomas-PCM (90 [34-205] vs. 152 [91-270], $p<0.001)$, y un intervalo PCM-apertura de la arteria ligeramente superior al grupo no-Cl (120 [92-153] vs. 106 [67-144], $\mathrm{p}<0.001$ ). Con respecto al tiempo total de isquemia (inicio de síntomas-apertura de arteria) el grupo $\mathrm{Cl}$ presentaba un tiempo inferior al grupo no- $\mathrm{Cl}(220$ [155-380] vs. 272 [196-456], $p=0.003)$, principalmente mediado por el menor tiempo inicio de síntomas-PCM. En la evaluación específica de los periodos observamos los distintos intervalos según el centro de proveniencia de las pacientes (figs. 1-3).

En cuanto al tratamiento recibido, el grupo $\mathrm{Cl}$ presentó mayor porcentaje de acceso radial en el grupo $\mathrm{Cl}(67.6 \%$ vs. $54.3 \%, p<0.001)$ y de implantación de endoprótesis farmacoactivas $(19.7 \%$ vs. $2.7 \%, p<0.001)$. 
Tabla 2 Procedencia, intervalos de tiempo y tratamiento

\begin{tabular}{|c|c|c|c|}
\hline Características & $\begin{array}{l}\text { Mujeres no- } \mathrm{Cl} \\
(\mathrm{n}=186)\end{array}$ & $\begin{array}{l}\text { Mujeres } \mathrm{Cl} \\
(\mathrm{n}=315)\end{array}$ & Valor de $\mathrm{p}$ \\
\hline Lugar del PCM, n (\%) & & & $<0.001$ \\
\hline Hospital con AP 24/7 & $155(83.3)$ & $50(15.9)$ & \\
\hline Hospital sin AP $24 / 7$ & $31(16.7)$ & $140(44.4)$ & \\
\hline SEM & $0(0)$ & $93(29.5)$ & \\
\hline$C A P$ & $0(0)$ & $32(10.2)$ & \\
\hline $\begin{array}{l}\text { Lugar del PCM fuera del } \\
\text { hospital con AP 24/7, } \\
\text { n (\%) }\end{array}$ & $31(16.7)$ & $265(84.1)$ & $<0.001$ \\
\hline \multicolumn{4}{|c|}{ Periodo (minutos), mediana (RIC) } \\
\hline $\begin{array}{l}\text { Inicio de } \\
\text { síntomas-PCM (min) }\end{array}$ & $152[91-270]$ & 90 [34-205] & $<0.001$ \\
\hline $\begin{array}{l}\text { PCM-apertura de } \\
\text { arteria (min) }\end{array}$ & $106[67-144]$ & $120[92-153]$ & $<0.001$ \\
\hline $\begin{array}{l}\text { Inicio de } \\
\text { síntomas-apertura de } \\
\text { arteria (tiempo total } \\
\text { de isquemia) (min) }\end{array}$ & $272[196-456]$ & $220[155-380]$ & 0.003 \\
\hline Acceso arterial, $n(\%)$ & & & $<0.001$ \\
\hline Femoral & $73(39.2)$ & $74(23.5)$ & \\
\hline Radial & $101(54.3)$ & $213(67.6)$ & \\
\hline Cruce radial-femoral & $12(6.5)$ & $28(8.9)$ & \\
\hline Anti-ıb/ıa, n (\%) & $56(30.3)$ & $80(25.4)$ & 0.237 \\
\hline$B C P I A o, n(\%)$ & $15(8.1)$ & $15(4.8)$ & 0.128 \\
\hline $\begin{array}{l}\text { Tratamiento recibido, } \\
n(\%)\end{array}$ & & & $<0.001$ \\
\hline Stent convencional & $160(86)$ & $205(65.1)$ & \\
\hline Stent farmacoactivo & $5(2.7)$ & $62(19.7)$ & \\
\hline Stent no implantado & $21(11.3)$ & $48(15.2)$ & \\
\hline
\end{tabular}

AP 24/7: servicio de angioplastia primaria $24 \mathrm{~h}$ al día 7 días a la semana; BCPIAo: balón de contrapulsación intraaórtico; CAP: centro de atención primaria; $\mathrm{Cl}$ : grupo posterior a la implantación del «Codi Infart»; no-Cl: grupo previo a la implantación del «Codi Infart»; PCM: primer contacto médico; RIC: rango intercuartílico; SEM: «Sistema d’Emergències Mèdiques».

\section{Resultados clínicos}

Los datos que comparan los resultados clínicos entre los grupos se presentan en la tabla 3.

No se observaron diferencias en la variable de valoración primaria (eventos cardiovasculares adversos mayores a 180 días) $(\mathrm{Cl}: 14.2 \%$ vs. no- $\mathrm{Cl}: 15.6 \%, \mathrm{p}=0.692)$, ni en las otras variables de valoración clínica.

\section{Discusión}

Las principales conclusiones de este estudio fueron que la implementación de la red de atención al IAMCEST: 1) ha aumentado la cantidad y el porcentaje de mujeres con IAMCEST tratadas mediante AP; 2) ha disminuido los tiempos totales de isquemia en población femenina; y 3) ha modificado el origen de las pacientes referidas para AP.

\section{Consideraciones generales de la introducción del «Codi Infart»}

La consecuencia más relevante de la implantación del $\mathrm{Cl}$ ha sido la generalización de la práctica de la AP como tratamiento de reperfusión del IAMCEST.
En la etapa previa a la implantación de la red, los pacientes del área geográfica dependiente de nuestra institución únicamente recibían AP como tratamiento en el IAMCEST cuando eran atendidos en primera instancia en nuestro centro o en caso de ser atendidos en otros centros hospitalarios si presentaban contraindicación para la trombolisis, requerían una angioplastia de rescate o se encontraban en choque cardiogénico. Tras la implantación del $\mathrm{Cl}$ todos los pacientes de nuestra área de referencia con IAMCEST son enviados a nuestro centro para la realización de AP, si el retraso estimado hasta la AP es inferior a $120 \mathrm{~min}$.

Este cambio ha sido posible gracias a la introducción de 2 elementos ${ }^{16,17}$ :

1 Activación a distancia de los equipos de hemodinámica de guardia. En la etapa anterior a la implantación de la red, la mayor parte de los pacientes de nuestra área quedaban excluidos del tratamiento de reperfusión mediante AP ya que eran atendidos en primer término en otras instituciones. En la etapa del $\mathrm{Cl}^{14}$, al transferir el poder de decisión en la activación de los equipos de hemodinámica a médicos de otros centros se ha producido una inversión en el origen de los pacientes. Si antes del $\mathrm{Cl}$ aproximadamente el $85 \%$ de los pacientes provenían de nuestro centro, y 
Tabla 3 Resultados clínicos

\begin{tabular}{|c|c|c|c|}
\hline Características & $\begin{array}{l}\text { Mujeres no-Cl } \\
(n=186)\end{array}$ & $\begin{array}{l}\text { Mujeres } \mathrm{Cl} \\
(\mathrm{n}=315)\end{array}$ & Valor de $p$ \\
\hline ECVM intrahospitalarios, $n(\%)$ & $25(13.4)$ & $32(10.2)$ & 0.264 \\
\hline Muerte global intrahospitalaria, $n$ (\%) & $23(12.4)$ & $28(8.9)$ & 0.214 \\
\hline Reinfarto intrahospitalario, n (\%) & $2(1.1)$ & $4(1.3)$ & 0.847 \\
\hline ACV intrahospitalario, $\mathrm{n}(\%)$ & $0(0)$ & $3(1)$ & 0.182 \\
\hline ECVM a 30 días, $n$ (\%) & $26(14.3)$ & $39(12.6)$ & 0.590 \\
\hline Muerte global a 30 días, $\mathrm{n}(\%)$ & $25(13.7)$ & $30(9.7)$ & 0.168 \\
\hline Reinfarto a 30 días, n (\%) & $2(1.1)$ & $7(2.3)$ & 0.354 \\
\hline ACV a 30 días, $n(\%)$ & $0(0)$ & $6(1.9)$ & 0.367 \\
\hline ECVM a 180 días, $n$ (\%) & $28(15.6 \%)$ & $44(14.2 \%)$ & 0.692 \\
\hline Muerte global a 180 días, $\mathrm{n}(\%)$ & $27(15.1)$ & $35(11.3)$ & 0.230 \\
\hline Reinfarto a 180 días, n (\%) & $2(1.1)$ & $9(2.9)$ & 0.198 \\
\hline ACV a 180 días, $\mathrm{n}(\%)$ & $0(0.0)$ & $6(1.9)$ & 0.061 \\
\hline
\end{tabular}

ACV: accidente cerebrovascular; Cl: grupo posterior a la implantación del «Codi Infart»; ECVM: eventos cardiovasculares adversos mayores; no-Cl: grupo previo a la implantación del «Codi Infart».

el $15 \%$ de otros hospitales, en la actualidad solo el $15 \%$ proviene de nuestra institución, mientras que el $85 \%$ restante procede de otros centros hospitalarios, de centros de atención primaria o son directamente transferidos por el «Sistema d'Emergències Mèdiques».

2 Coordinación de la asistencia médica por el «Sistema d'Emergències Mèdiques». La centralización de la asistencia por el Centro Coordinador del «Sistema d'Emergències Mèdiques» ${ }^{14}$ permitió el contacto de nuestro centro con los otros agentes involucrados en la atención de las pacientes, y posibilitó conocer las características clínicas y los tiempos estimados de llegada de las pacientes para

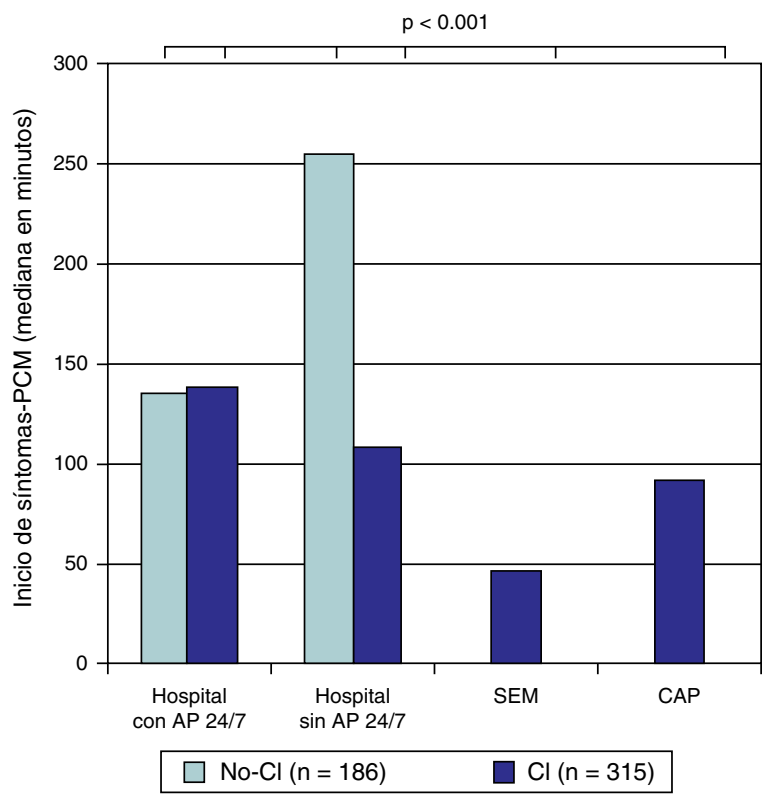

Figura 1 Periodo inicio de síntomas-PCM según la procedencia. AP 24/7: servicio de angioplastia primaria $24 \mathrm{~h}$ al día 7 días a la semana; CAP: centro de atención primaria; $\mathrm{Cl}$ : grupo posterior a la implantación del «Codi Infart»; no-Cl: grupo previo a la implantación del «Codi Infart»; PCM: primer contacto médico; SEM: «Sistema d'Emergències Mèdiques». organizar eficazmente la realización de la AP en nuestro centro, así como facilitar el retorno a los hospitales de referencia.

\section{Variaciones en el número y porcentaje de mujeres atendidas}

Como consecuencia de la expansión de los centros proveedores, se ha producido un aumento espectacular de la tasa de mujeres tratadas mediante AP, la cual se ha multiplicado 5 veces pasando de 2.09 casos/mes a $10.16 \mathrm{casos} / \mathrm{mes}$.

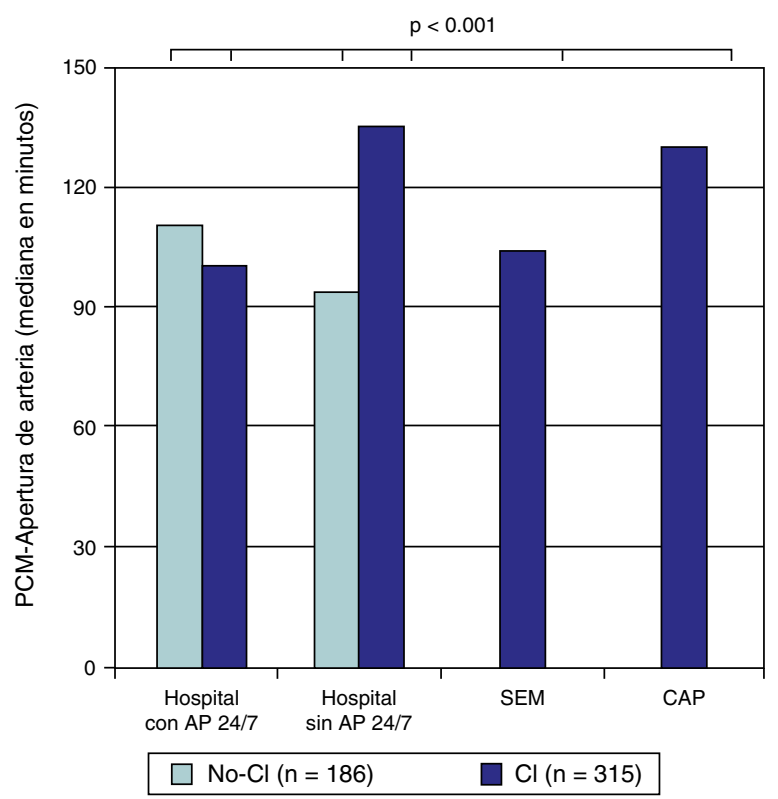

Figura 2 Periodo PCM-apertura de arteria según la procedencia. AP 24/7: servicio de angioplastia primaria $24 \mathrm{~h}$ al día 7 días a la semana; CAP: centro de atención primaria; $\mathrm{Cl}$ : grupo posterior a la implantación del «Codi Infart»; no-Cl: grupo previo a la implantación del «Codi Infart»; PCM: primer contacto médico; SEM: «Sistema d'Emergències Mèdiques». 


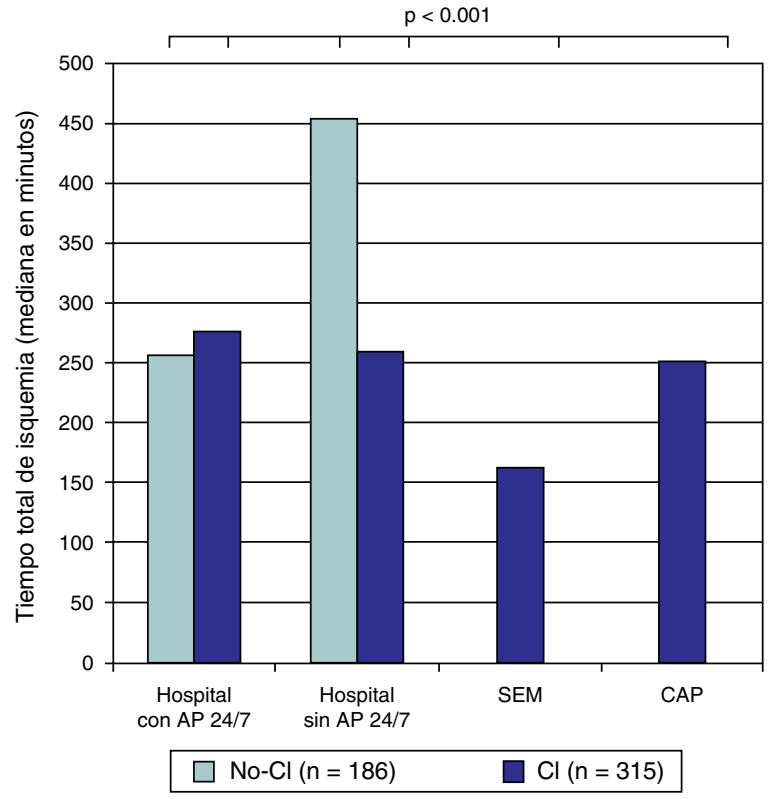

Figura 3 Tiempo total de isquemia según la procedencia. AP 24/7: servicio de angioplastia primaria $24 \mathrm{~h}$ al día 7 días a la semana; CAP: centro de atención primaria; $\mathrm{Cl}$ : grupo posterior a la implantación del «Codi Infart»; no-Cl: grupo previo a la implantación del «Codi Infart»; SEM: «Sistema d'Emergències Mèdiques».

Aparte del aumento general de AP realizadas en mujeres, también se ha producido un incremento en el porcentaje de las mismas con respecto al total de pacientes $(18.5 \%$ vs. $22.2 \%, p=0.028$ ). Este incremento más acusado en el género femenino podría ser debido a que la implantación del $\mathrm{Cl}$ ha permitido mejorar la sensibilidad en el diagnóstico del IAMCEST, especialmente entre las mujeres.

\section{Reducción de los intervalos de atención médica}

El tiempo total de isquemia, que comprende el periodo desde que se inician los síntomas hasta que el paciente recibe la terapia de reperfusión, es el tiempo mejor relacionado con la mortalidad y la discapacidad a largo plazo ${ }^{18}$. Estudios previos han demostrado que las mujeres presentan mayores tiempos totales de isquemia, debido a retrasos en acceder al sistema médico, en el diagnóstico y en el tratamiento ${ }^{13,19,20}$.

En nuestro estudio se observa cómo tras la implantación del $\mathrm{Cl}$ se produjo una drástica reducción del tiempo total de isquemia (inicio de síntomas-apertura de arteria) (220 min vs. 272 minu, $p=0.003$ ). Esto se produjo principalmente a expensas de un reducción en el intervalo inicio de síntomasPCM $(90 \mathrm{~min}$ vs. $152 \mathrm{~min}, \mathrm{p}<0.001)$, ya que el intervalo PCM-apertura de arteria presentó un pequeño incremento (120 min vs. $106 \mathrm{~min}, \mathrm{p}<0.001$ ).

El intervalo inicio de síntomas-PCM de las pacientes atendidas en nuestra institución antes y después del $\mathrm{Cl}$ se ha mantenido estable. La principal diferencia ha venido mediada por una mejoría en el intervalo inicio de síntomasPCM de las pacientes provenientes de hospitales sin AP 24/7, de centros de atención primaria y especialmente del «Sistema d'Emergències Mèdiques», esto último debido a la rápida movilización de este servicio ante la sospecha de un IAMCEST.

Aunque la implantación del $\mathrm{Cl}$ ha supuesto una mejor organización, es sorprendente observar cómo en nuestro trabajo el intervalo PCM-apertura de arteria aumentó ligeramente. Este dato hay que explicarlo debido a que tras la implantación del sistema de atención en red al infarto (grupo $\mathrm{Cl}$ ), la gran mayoría de las activaciones del equipo de hemodinámica se producía cuando los pacientes se encontraban en centros sin AP 24/7, en centros de atención primaria o en su domicilio. $Y$ gran parte del tiempo consumido entre el PCM y la apertura de la arteria era debido a que los traslados extrahospitalarios requieren más tiempo que los traslados intrahospitalarios. Incluso, a pesar de este hecho, nuestros tiempos totales de isquemia han presentado una clara mejoría desde la instauración del $\mathrm{Cl}$, siendo similares a los reportados por otros grupos españoles ${ }^{9-11}$.

Asimismo, hay que resaltar que estudios recientes han demostrado que el traslado directo de los pacientes, sin necesidad de ingresar en los departamentos de urgencias, ha permitido disminuir los periodos y mejorar el pronóstico de los mismos ${ }^{21-23}$. Estos datos indican que se deben implementar mecanismos para generalizar la atención precoz mediante los sistemas de emergencias y el traslado directo de las pacientes a los laboratorios de hemodinámica.

\section{Manejo médico y pronóstico}

Tradicionalmente, la población femenina ha recibido un tratamiento más conservador, debido a la administración en menor medida de los tratamientos médicos recomendados y un menor acceso a la $\mathrm{AP}^{13}$. $\mathrm{El} \mathrm{Cl}$, al tener un protocolo de actuación estandarizado ha permitido uniformizar el tratamiento en ambos sexos, a nivel intervencionista y de tratamiento antitrombótico. También observamos otras mejoras en el tratamiento, como el mayor uso del acceso radial y de endoprótesis farmacoactivas, relacionados con la progresiva implantación en la clínica habitual de la evidencia generada en los últimos tiempos ${ }^{24-26}$.

En cuanto al pronóstico de las mujeres evaluadas en nuestro estudio, no se observaron diferencias significativas entre los 2 periodos evaluados. La relativamente pequeña cantidad de pacientes incluidos y el seguimiento realizado únicamente a medio plazo (180 días) en nuestro estudio podría haber impedido detectar diferencias en el pronóstico entre ambos grupos, a pesar de la importante reducción en los tiempos totales de isquemia. Asimismo, dado que únicamente disponemos de datos de las pacientes referidas para AP, no se puede conocer exactamente la importancia pronóstica total de la instauración del $\mathrm{Cl}$. Sin embargo, dada la elevada mortalidad de las pacientes que no reciben tratamiento de reperfusión, nos hace hipotetizar que la instauración del sistema de atención en red al IAMCEST ha podido disminuir de modo importante la mortalidad en la población femenina, a tenor del importantísimo aumento de la tasa mensual de AP realizadas en mujeres ${ }^{11,27,28}$.

\section{Limitaciones}

Primero, este estudio es un análisis observacional y unicéntrico, y, aunque se realizó una recogida prospectiva de las 
variables, algunas diferencias pueden no haber sido detectadas y condicionar los resultados. Cabe destacar que los grupos de comparación pudieran ser más homogéneos si durante el primer periodo temporal se hubiesen incluido datos de todos los centros de nuestra área geográfica de referencia, y no únicamente de nuestra institución. Sin embargo, nuestro estudio es un análisis de un largo periodo y supone una buena representación de cómo ha variado la asistencia de las mujeres a lo largo del tiempo. Segundo, se desconocen la incidencia real de las pacientes con IAMCEST que reciben tratamiento trombolítico eficaz y por ello la interpretación de los datos debe ser cuidadosa. Sin embargo, la realización de trombolisis en nuestro medio es marginal ${ }^{9}$ y por ello nuestro trabajo representa a la población femenina que recibe tratamiento de reperfusión. Tercero, los datos se refieren solo a la población atendida en nuestra área de referencia, y por ende los resultados no se pueden extrapolar completamente a otras áreas geográficas.

\section{Conclusiones}

La implantación del $\mathrm{Cl}$ ha permitido aumentar notablemente la cantidad y porcentaje de mujeres con IAMCEST tratadas mediante AP en la fase aguda, así como reducir el tiempo total de isquemia. No se detectaron diferencias en cuanto al pronóstico, probablemente debido al reducido tamaño de la muestra y a la evaluación únicamente a medio plazo.

\section{Responsabilidades éticas}

Protección de personas y animales. Los autores declaran que los procedimientos seguidos se conformaron a las normas éticas del comité de experimentación humana responsable y de acuerdo con la Asociación Médica Mundial y la Declaración de Helsinki..

Confidencialidad de los datos. Los autores declaran que han seguido los protocolos de su centro de trabajo sobre la publicación de datos de pacientes.

Derecho a la privacidad y consentimiento informado. Los autores han obtenido el consentimiento informado de los pacientes y/o sujetos referidos en el artículo. Este documento obra en poder del autor de correspondencia.

\section{Financiación}

No se recibió patrocinio de ningún tipo para llevar a cabo este artículo.

\section{Conflicto de intereses}

Los autores declaran no tener ningún conflicto de intereses.

\section{Bibliografía}

1. Steg PG, James SK, Atar D, et al. ESC Guidelines for the management of acute myocardial infarction in patients presenting with ST-segment elevation. Eur Heart J. 2012;33:2569-619.
2. Heras M, Marrugat J, Aros F, et al. Reduction in acute myocardial infarction mortality over a five-year period. Rev Esp Cardiol. 2006;59:200-8.

3. Bairey Merz N, Bonow RO, Sopko G, et al. Women's Ischemic Syndrome Evaluation: Current status and future research directions: report of the National Heart, Lung and Blood Institute workshop: October 2-4, 2002: executive summary. Circulation. 2004;109:805-7.

4. Mehilli J, Kastrati A, Dirschinger J, et al. Sex-based analysis of outcome in patients with acute myocardial infarction treated predominantly with percutaneous coronary intervention. JAMA. 2002;287:210-5.

5. Hochman JS, Tamis JE, Thompson TD, et al. Sex, clinical presentation, and outcome in patients with acute coronary syndromes. Global Use of Strategies to Open Occluded Coronary Arteries in Acute Coronary Syndromes Ilb Investigators. N Engl J Med. 1999;341:226-32.

6. Benamer H, Tafflet M, Bataille S, et al., CARDIO-ARHIF Registry Investigators. Female gender is an independent predictor of in-hospital mortality after STEMI in the era of primary $\mathrm{PCl}$ : Insights from the greater Paris area PCI Registry. Eurolntervention. 2011;6:1073-9.

7. Widimský P, Budesínský T, Vorác D, et al., 'PRAGUE' Study Group Investigators. Long distance transport for primary angioplasty vs immediate thrombolysis in acute myocardial infarction. Final results of the randomized national multicentre trial-PRAGUE-2. Eur Heart J. 2003;24:94-104.

8. Regueiro A, Goicolea J, Fernández-Ortiz Macaya C, et al. STEMI Interventions: The European perspective and Stent for Life Initiative. Intervent Cardiol Clin. 2012;1:6.

9. Regueiro A, Tresserras R, Goicolea J, et al. Primary percutaneous coronary intervention: Models of intervention in Spain. Eurolntervention. 2012;8:4.

10. Barge-Caballero E, Vázquez-Rodríguez JM, Estévez-Loureiro R, et al. Primary angioplasty in Northern Galicia: Care changes and results following implementation of the PROGALIAM protocol. Rev Esp Cardiol (Engl Ed). 2012;65:341-9.

11. Gómez-Hospital JA, Dallaglio PD, Sánchez-Salado JC, et al. Impact on delay times and characteristics of patients undergoing primary percutaneous coronary intervention in the southern metropolitan area of Barcelona after implementation of the infarction code program. Rev Esp Cardiol (Engl Ed). 2012;65:911-8.

12. Hailer B, Naber C, Koslowski B, et al. Gender-related differences in patients with ST-elevation myocardial infarction: Results from the registry study of the ST elevation myocardial infarction network Essen. Clin Cardiol. 2011;34:294-301.

13. Greenberg MR, Miller AC, Mackenzie RS, et al. Analysis of sex differences in preadmission management of ST-segment elevation (STEMI) myocardial infarction. Gend Med. 2012;9:329-34.

14. Sectorizació de l'atenció a les persones malaltes amb infart agut de miocardi (IAM) amb elevació del segment, ST., per tal de portar a terme l'angioplàstia, primària., Instrucció 04/2009. CatSalut. 2009:1-7.

15. Tubaro M, Danchin N, Goldstein P, et al. Pre-hospital treatment of STEMI patients. A scientific statement of the Working Group Acute Cardiac Care of the European Society of Cardiology. Acute Cardiac Care. 2011;13:56-67.

16. Bradley EH, Curry LA, Webster TR, et al. Achieving rapid doorto-balloon times: How top hospitals improve complex clinical systems. Circulation. 2006;113:e1079-85.

17. Bradley EH, Herrin J, Wang Y, et al. Strategies for reducing the door-to-balloon time in acute myocardial infarction. N Engl J Med. 2006;355:23082320.

18. Gersh BJ, Stone GW, White HD, et al. Pharmacological facilitation of primary percutaneous coronary intervention for acute myocardial infarction: Is the slope of the curve the shape of the future? JAMA. 2005;293:979-86. 
19. Vaccarino V, Krumholz HM, Berkman LF, et al. Sex differences in mortality after myocardial infarction. Is there evidence for an increased risk for women? Circulation. 1995;91:1861-71.

20. Milcent C, Dormont B, Durand-Zaleski I, et al. Gender differences in hospital mortality and use of percutaneous coronary intervention in acute myocardial infarction: Microsimulation analysis of the 1999 nationwide French hospitals database. Circulation. 2007;115:833-9.

21. Estévez-Loureiro R, Calviño-Santos R, López-Sainz A, et al. Long-term prognostic benefit of field triage and direct transfer of patients with ST-segment elevation myocardial infarction treated by primary percutaneous coronary intervention. Am J Cardiol. 2013;111:1721-6.

22. Ortolani P, Marzocchi A, Marrozzini C, et al. Clinical impact of direct referral to primary percutaneous coronary intervention following pre-hospital diagnosis of ST-elevation myocardial infarction. Eur Heart J. 2006;27:15501557.

23. Pedersen SH, Galatius S, Hansen PR, et al. Field triage reduces treatment delay and improves long-term clinical outcome in patients with acute ST-segment elevation myocardial infarction treated with primary percutaneous coronary intervention. J Am Coll Cardiol. 2009;54:22962302.
24. Mehta SR, Jolly SS, Cairns J, et al., RIVAL Investigators. Effects of radial versus femoral artery access in patients with acute coronary syndromes with or without ST-segment elevation. J Am Coll Cardiol. 2012;60:2490-9.

25. Sabate M, Cequier A, Iñiguez A, et al. Everolimus-eluting stent versus bare-metal stent in ST-segment elevation myocardial infarction (EXAMINATION): 1 year results of a randomised controlled trial. Lancet. 2012;380:1482-90.

26. Räber L, Kelbak H, Ostojic $M$, et al., COMFORTABLE AMI Trial Investigators. Effect of biolimus-eluting stents with biodegradable polymer vs bare-metal stents on cardiovascular events among patients with acute myocardial infarction: the COMFORTABLE AMI randomized trial. JAMA. 2012;308: 777-87.

27. Wood FO, Leonowicz NA, Vanhecke TE, et al. Mortality in patients with ST-segment elevation myocardial infarction who do not undergo reperfusion. Am J Cardiol. 2012;110: 509-14.

28. Figueras J, Heras M, Baigorri F, et al. III Catalan registry of ST elevation acute myocardial infarction. Comparison with former Catalan registries I and II from Catalonia, Spain. Med Clin (Barc). 2009;133:694-701. 